\title{
Phase shift of amplitude-modulated optical signals in graphene oxide water dispersions due to thermal lens focal length oscillation
}

\author{
Sonia Melle, ${ }^{1, *}$ Oscar G. Calderón, ${ }^{1}$ Ana Egatz-Gómez, ${ }^{2,1}$ E. Cabrera-Granado, ${ }^{1}$ \\ F. Carreño, ${ }^{1}$ M. A. Antón ${ }^{1}$ and H. J. Salavagione ${ }^{3}$ \\ ${ }^{1}$ Departamento de Óptica, Universidad Complutense de Madrid, Arcos de Jalón 118, \\ Madrid 2803\%, Spain \\ ${ }^{2}$ Department of Chemical and Biomolecular Engineering, University of Notre Dame, Notre \\ Dame, Indiana 46556, USA \\ 3 Departamento de Física de Polímeros, Elastómeros y Aplicaciones Energéticas, Instituto \\ de Ciencia y Tecnología de Polímeros, CSIC, Juan de la Cierva 3, Madrid 28006, Spain \\ *Corresponding author: smelle@fis.ucm.es
}

\begin{abstract}
We analyze the phase shift induced in an amplitude-modulated laser beam propagating through a water dispersion of graphene oxide sheets in a fiber-to-fiber U-bench. This phase shift arises from the thermally induced nonlinear refraction in the sample. The system exhibits strong optical limiting performance for weak continuous-wave signals. A theoretical model including beam propagation and thermal lens focal length oscillation reproduces the experimental findings. (c) 2015 Optical Society of America
\end{abstract}

OCIS codes: $190.4370,350.6830$.

\section{Introduction}

Graphene oxide (GO) can be synthesized by chemical oxidation of graphite [1]. The graphenederived sheets in graphite oxide (graphene oxide flakes) present oxygen-containing functional groups that renders GO a good candidate among materials with tunable optical properties through chemical functionalization [2]. The presence of these functional groups makes GO sheets strongly hydrophilic and dispersible in water [3-5]. This particular feature has increasingly attracted the interest of researchers on applications of GO in optoelectronics and biomedicine (see review by Loh et al. [2]). 
GO dispersions exhibit broadband nonlinear optical properties in the nanosecond, picosecond, and femtosecond time regimes [6-13]. A saturable absorption behavior was found in $\mathrm{GO}$ aqueous dispersions under $16 \mathrm{GW} / \mathrm{cm}^{2}$, 80 fs laser pulses at $790 \mathrm{~nm}$ [6]. GO N,Ndimethylformamide (DMF) dispersions also showed a saturable absorption behavior under $2.1 \mathrm{GW} / \mathrm{cm}^{2}, 35 \mathrm{ps}$ pulses at $532 \mathrm{~nm}$; while at higher intensities $\left(>4.5 \mathrm{GW} / \mathrm{cm}^{2}\right)$, two photon absorption was found to dominate the nonlinear absorption [7]. In the nanosecond regime, excited state absorption contributed to the nonlinear absorption with a saturation intensity of $0.12 \mathrm{GW} / \mathrm{cm}^{2}[7]$.

GO nanosheets dispersions in organic-solvents and water under visible and infrared nanosecond and picosecond laser pulses exhibit strong optical limiting (OL) performance [8-10]. OL is a nonlinear optical process in which the transmittance of a material decreases as the input light intensity increases, and it can be used to fabricate devices for protecting eyes and sensors from powerful laser beams. In graphene nanosheets organic dispersions under nanosecond laser pulses, the responsible mechanism for OL is nonlinear scattering from vapor bubbles forming around the graphene flakes, and the surface tension of the solvents determines the effectiveness of the OL effect [8]. It was also found that the OL response is stronger under visible than under infrared nanosecond laser pulses $[8,9]$ and that the reduced graphene nanosheets exhibit enhanced OL behavior compared to their GO precursors [9]. An improvement of the OL performance of graphene oxide dispersions has been achieved by linking the graphene nanosheets with nonlinear optical molecules [11].

In previously mentioned works the nonlinear behavior arises at intensities in the order of $\mathrm{GW} / \mathrm{cm}^{2}$. On the contrary, at low input intensities and continuous-wave operation different non-linear mechanisms can become evident. For example, spatial self-phase modulation was reported in graphene sheets organic dispersions due to a huge broadband third-order optical nonlinearity [12]. In the quasicontinuous regime, Li et al. [14] reported negative time delays for amplitude-modulated beams propagating through single-layer GO aqueous dispersions under visible illumination. The authors attributed the time delays to the GO dispersion reversible saturable absorption. However, in the quasicontinuous regime or for long duty cycle operation, heating can significantly change the local medium density and thermal effects become relevant. In fact, transient thermal effects have been observed in DMF GO dispersions by Zhang et al. [13], even up to the nanosecond regime.

In this work we focus our attention on the propagation of a low-frequency amplitudemodulated infrared laser beam through GO aqueous dispersions. We analyze the thermally induced time delay between the signal propagated through the GO dispersion and the signal propagating through a deionized water sample. We used a fiber-to-fiber U-bench setup to study the time delay and its dependence with GO concentration, optical input power and modulation frequency. Transmittance measurements show an OL behavior, which agrees with 
the negative phase delays measured. Our simple theoretical model includes beam propagation and thermal lens effects, and reproduces our experimental findings, which suggests that thermally induced nonlinear refraction in the GO dispersion is the main responsible for the measured phase shifts.

\section{Experimental setup}
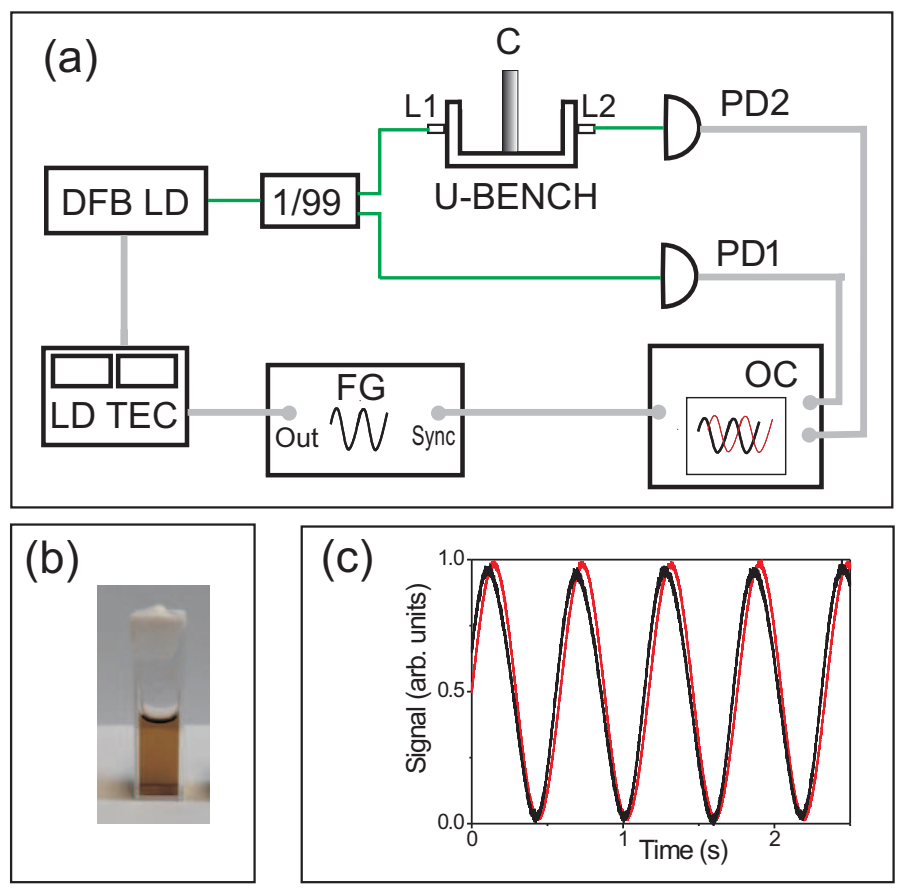

Fig. 1. (a) Experimental setup. C, cuvette containing the graphene oxide dispersion. DFB LD, distributed feedback laser diode at $977 \mathrm{~nm}$. LD TEC, laser diode temperature and current controller. FG, function generator. OC, digital oscilloscope. PD1, reference photodetector. PD2, photodetector for the GO signal. L1, collimating lens. L2, focusing lens. Green lines, single mode optical fibers. (b) Cuvette containing an aquoeus dispersion of GO sheets with a concentration of $0.25 \mathrm{mg} / \mathrm{mL}$. (c) Time evolution of the experimental reference signal (red line) and the signal propagated through the GO dispersion and the U-bench (black line) for a $0.5 \mathrm{mg} / \mathrm{mL}$ graphene oxide water dispersion, a laser input power of $P_{0}=28.5 \mathrm{~mW}$, and a modulation frequency of $f_{m}=1.71 \mathrm{~Hz}$.

A $4 \mathrm{mg} / \mathrm{mL}$ GO water dispersion was supplied by Graphenea (Spain). The dispersion exhibited long-term stability. According to the manufacturer, the dispersion is made of sheets with dimensions ranging from a few hundred nanometers to a few micrometers (TEM, not 
shown), the monolayer content is greater than 95\%, and it has a considerable degree of oxidation from C-O epoxy/ether functional groups (C1s XPS, not shown), and a C:O ratio of $1.4: 1$. For our experiments we diluted the commercial dispersion to concentrations from 0.25 to $1 \mathrm{mg} / \mathrm{mL}$.

To study the time delay induced in an amplitude-modulated laser beam propagating through the GO water dispersion we used the fiber-based experimental setup depicted in Fig. 1(a). A quartz cuvette (Hellma, 100-QS) with a $10 \mathrm{~mm}$ path length (see Fig. 1(b)) was placed in the center of a fiber-to-fiber U-bench (Thorlabs, FBC-1550-APC). The U-bench had a free-space length of $59.8 \mathrm{~mm}$, and two glued AR-coated aspheric lenses for light collimation and coupling. According to the manufacturer, the typical collimated beam diameter is $3.1 \mathrm{~mm}$ (at $1550 \mathrm{~nm}$ ).

The laser beam comes from a pigtailed laser diode (Thorlabs, PL975P200) operating at $977 \mathrm{~nm}$. The laser was held at room temperature using a current and temperature feedback control module (Thorlabs, ITC510). The light beam was split into two. One percent of the beam (1\%) was launched directly into a switchable-gain amplified InGaAs photodetector (Thorlabs, PDA10CS-EC), which was used as reference (PD1 in Fig. 1(a)). The other part of the beam (99\%) was launched onto the GO dispersion under study and then to an identical photodetector (PD2 in Fig. 1(a)). We recorded two signals in a digital oscilloscope (Agilent, DSO9104A), the reference signal and the signal interacting with the graphene oxide dispersion. The two recorded signals were averaged over 16 or 64 oscilloscope traces using a synchronous output signal from a function generator as a trigger.

The injection current to the laser beam was sinusoidally modulated using a function generator (Agilent, 33220A). As a result, the laser power was modulated according to $P=P_{0}+P_{m} \cos \left(2 \pi f_{m} t\right)$, where $P_{0}$ is the average power, $P_{m}$ is the modulation amplitude, and $f_{m}$ is the modulation frequency. We used a modulation amplitude ratio of $P_{m} / P_{0}=0.15$ in all the experiments reported in this work. As an example, in Fig. 1(c) we plot the time evolution of both, the reference signal (red line) and the GO signal (black line), for a 0.5 $\mathrm{mg} / \mathrm{mL}$ GO water dispersion, a laser input power of $P_{0}=28.5 \mathrm{~mW}$, and a modulation frequency of $f_{m}=1.71 \mathrm{~Hz}$. Here the GO signal has a negative phase shift relative to the reference.

In order to obtain the phase shift between both signals we perform the correlation between them by using a deionized water cuvette to set the zero phase shift of our system. For comparison purposes, we also measure the input-output laser power curve using a continuouswave laser beam, and a power meter (Thorlabs, PM122). 


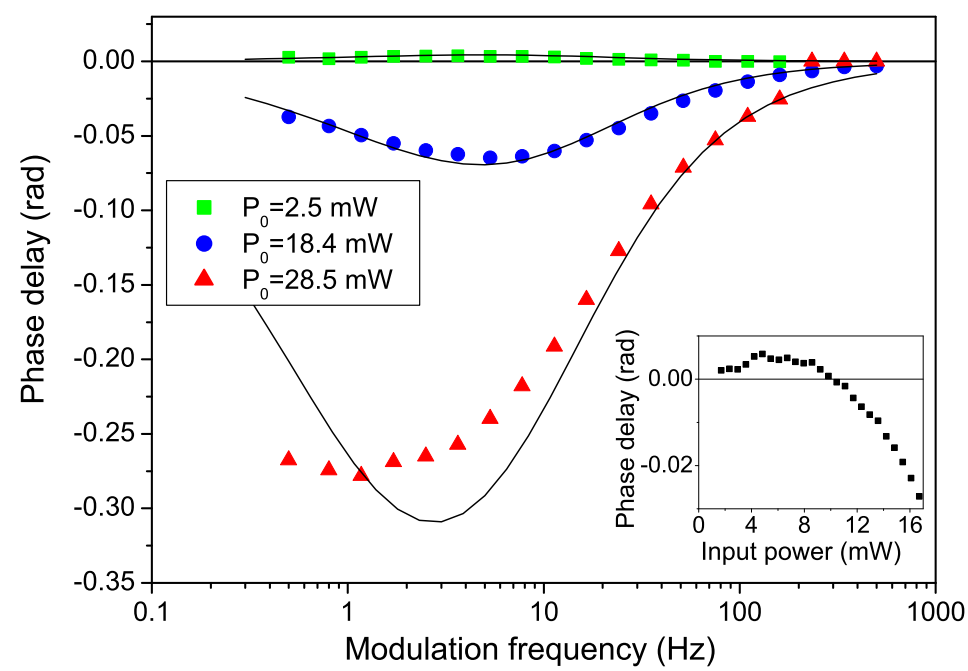

Fig. 2. Experimental (symbols) and theoretical (lines) phase delay versus modulation frequency for a $0.5 \mathrm{mg} / \mathrm{mL}$ GO aqueous dispersion and different input powers: $P_{0}=2.5 \mathrm{~mW}$ (squares), $P_{0}=18.4 \mathrm{~mW}$ (circles), $P_{0}=28.5 \mathrm{~mW}$ (triangles). Inset: phase delay versus input power for a modulation frequency of $f_{m}=3.64 \mathrm{~Hz}$.

\section{Experimental results}

\section{A. Phase shift results}

We analyzed the phase shift of the amplitude-modulated laser beam propagating through the GO water dispersion relative to the reference as a function of the modulation frequency and the GO concentration.

We measured the phase shift for modulation frequencies $f_{m}$ from 0.5 to $500 \mathrm{~Hz}$ in a 0.5 $\mathrm{mg} / \mathrm{mL}$ GO aqueous dispersion. Figure 2 shows the experimental results for different input powers: $P_{0}=2.5 \mathrm{~mW}$ (squares), $P_{0}=18.4 \mathrm{~mW}$ (circles), and $P_{0}=28.5 \mathrm{~mW}$ (triangles). The phase shift becomes large enough to be measured at modulation frequencies smaller than 1 $\mathrm{kHz}$, which implies the characteristic time of the phenomenon underlying these phase delays is in the order of the milliseconds to tenths of a second. The maximum phase shift is power dependent; it changes sign with input power and occurs at a modulation frequency between 1 and $10 \mathrm{~Hz}$. For an input power of $P_{0}=2.5 \mathrm{~mW}$, phase delays are positive, whereas higher input powers lead to negative phase delays, for all the modulation frequencies within our experimental range. For an input power of $P_{0}=28.5 \mathrm{~mW}$, the maximum phase shift is -0.3 


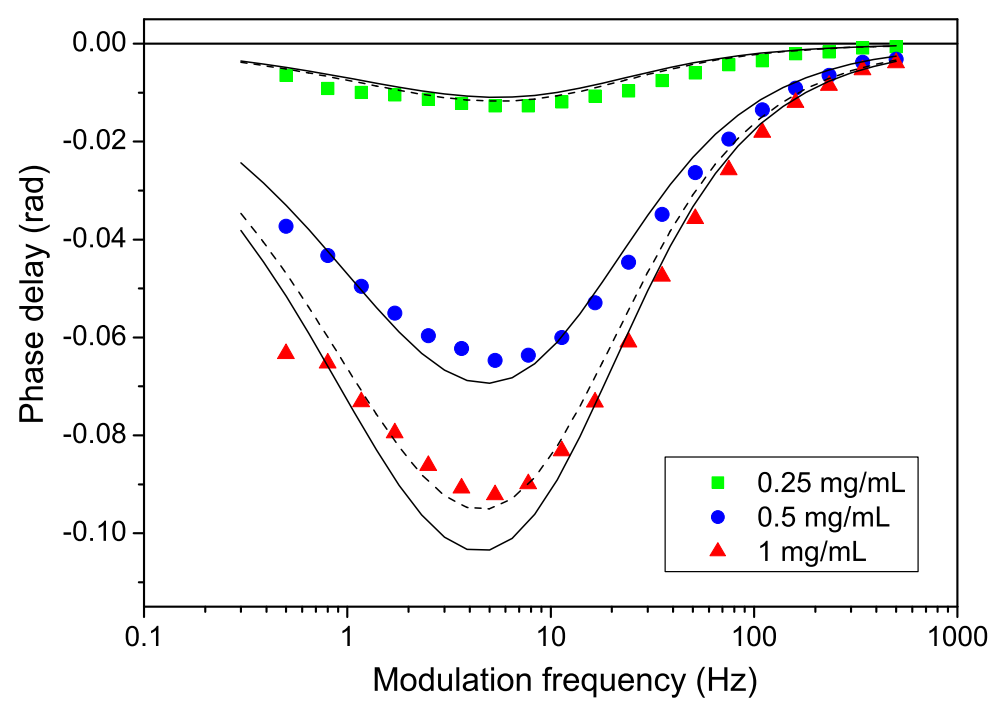

Fig. 3. Experimental (symbols) and theoretical (lines) phase delay versus modulation frequency for a laser input power of $18.4 \mathrm{~mW}$ and different $\mathrm{GO}$ concentrations: $0.25 \mathrm{mg} / \mathrm{mL}$ (squares), $0.5 \mathrm{mg} / \mathrm{mL}$ (circles), and $1 \mathrm{mg} / \mathrm{mL}$ (triangles). Simulated curves with a fixed value of the thermal conductivity $\kappa$ (solid lines), and changing the value of $\kappa$ according to the volume fraction of GO sheets (dashed lines).

rad at a modulation frequency of approximately $2 \mathrm{~Hz}$. The sign change of the phase shift with input power can be clearly seen in Fig. 2 inset where we plotted the phase shift versus the input power for a modulation frequency $f_{m}=3.64 \mathrm{~Hz}$. These results indicate that the GO dispersion can be used to control the phase of the amplitude-modulated beam.

We also studied the phase shift dependence with GO concentration. In Fig. 3 we plot this magnitude as a function of the modulation frequency $f_{m}$ for an input power $P_{0}=18.4$ $\mathrm{mW}$ and GO concentrations of: $0.25 \mathrm{mg} / \mathrm{mL}$ (squares), $0.5 \mathrm{mg} / \mathrm{mL}$ (circles), and $1 \mathrm{mg} / \mathrm{mL}$ (triangles). We observe an overall enhancement of the phase shift as the GO concentration increases.

\section{B. Transmittance measurements}

To investigate the mechanisms underlying the phase shift induced by the GO dispersion, first we studied the GO transmittance for a continuous-wave laser beam. We measured the input power at the cuvette entrance and the output power at the end of the U-bench output optical fiber. Figure 4(a) shows the output-input power curves for $0.25 \mathrm{mg} / \mathrm{mL}, 0.5 \mathrm{mg} / \mathrm{mL}$, 

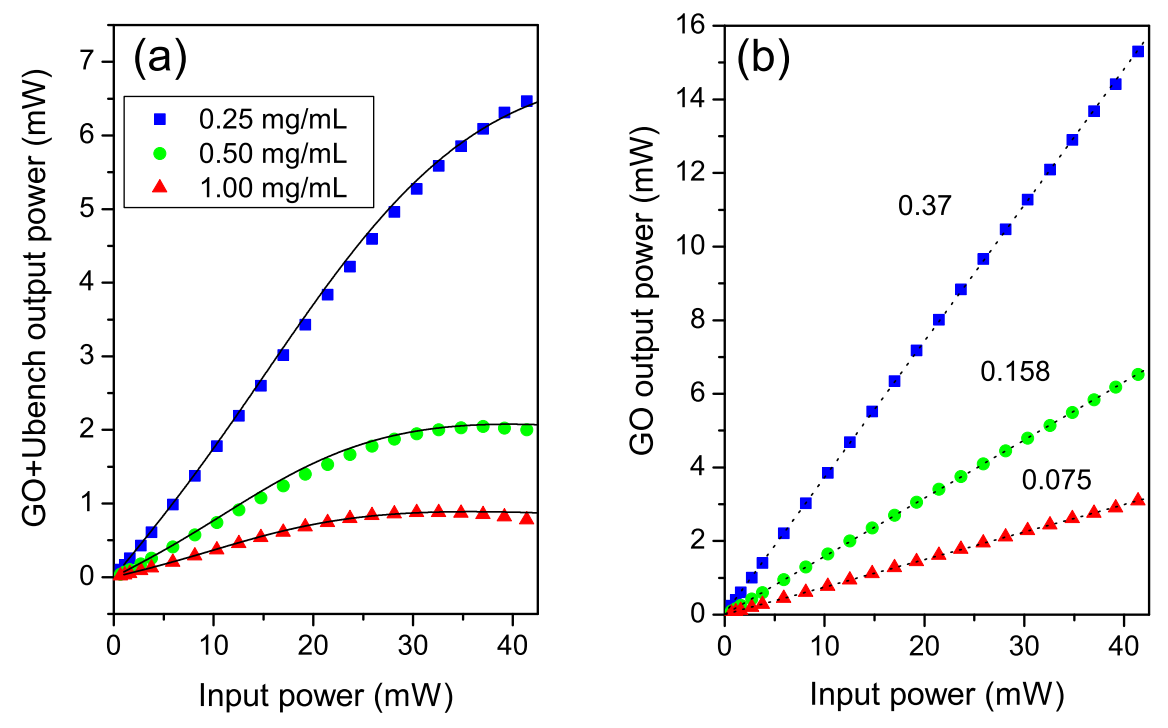

Fig. 4. Experimental (symbols) output-input power curves for different GO concentrations: $0.25 \mathrm{mg} / \mathrm{mL}$ (squares), $0.5 \mathrm{mg} / \mathrm{mL}$ (circles), and $1 \mathrm{mg} / \mathrm{mL}$ (triangles). (a) Output power measured at the output optical fiber of the Ubench. Theoretical curves (solid lines). (b) Output power measured at the output of the cuvette (before the beam enters L2). The dotted lines are linear fits to the experimental data and the corresponding slopes are also indicated in panel (b).

and $1 \mathrm{mg} / \mathrm{mL}$ GO dispersions. We see that the overall transmission decreases as the input power increases. That is, a strong OL performance is obtained. The OL threshold, defined as the input power at which the transmittance falls to the $50 \%$ of the value at very low powers, is around $45 \mathrm{~mW}$.

To gain a deeper insight into the origin of the phase delay and the OL behavior in our particular case we examine our experimental system in detail. The U-bench has two optical lenses of fixed focal length: lens L1 collimates the light coming from the input fiber into the cuvette, and lens L2 focuses the beam coming from the cuvette onto the output fiber. Thus, the output power shown in Fig. 4(a) is measured after the beam is transmitted through the GO dispersion, L2, and the U-bench output optical fiber. In order to isolate the role played by the GO dispersion in the OL behavior we measured the power of the beam coming out of the cuvette, before it enters L2. These results are shown in Fig. 4(b). A linear response is obtained in the whole input power range which reveals a linear absorption regime occurs in the aqueous GO dispersions. This is an expected result if we consider that the intensities used in our 
experiments are below $1 \mathrm{~W} / \mathrm{cm}^{2}$ (a $1.55 \mathrm{~mm}$ beam radius with $50 \mathrm{~mW}$ power corresponds to an intensity of $\left.0.66 \mathrm{~W} / \mathrm{cm}^{2}\right)$. This value is much lower than the typical nonlinear absorption intensities used in studies of OL by GO nanostructures under ns pulses, which is in the order of $\mathrm{MW} / \mathrm{cm}^{2}$ [9]. Using the linear fit slope of Fig. 4(b) and taking into account the transmittance of the cuvette $\left(T^{\text {cuvette }} \simeq 0.93\right.$ ), we obtained an effective linear absorption coefficient of the GO samples of $\alpha_{\text {eff }}=0.92 \mathrm{~cm}^{-1}(0.25 \mathrm{mg} / \mathrm{mL}), \alpha_{\text {eff }}=1.77 \mathrm{~cm}^{-1}(0.5$ $\mathrm{mg} / \mathrm{mL})$, and $\alpha_{e f f}=2.52 \mathrm{~cm}^{-1}(1 \mathrm{mg} / \mathrm{mL})$. These values are in agreement with values found in other works [16]. This result rules out the possibility that nonlinear absorption takes place in the sample. In our case, the phase shift observed in our sub-W/cm2, $977 \mathrm{~nm}$ amplitude modulated laser beam can be attributed neither to reversible saturable absorption nor to free-carrier absorption $[8,14,17,18]$. In fact, the phase shift induced by reversible saturable absorption $[14,19,20]$ or by free carrier absorption [21] for amplitude-modulated signals can be estimated as:

$$
\phi \simeq-\alpha_{0} L \frac{I_{0}}{I_{\text {sat }}} \frac{2 \pi f_{m} \tau_{c}}{1+\left(2 \pi f_{m} \tau_{c}\right)^{2}}
$$

Here $\alpha_{0}$ is the effective linear absorption coefficient, and $L$ is the sample length. In our case, the optical density is around unity, i.e. $\alpha_{0} L \simeq 1$. The laser intensity $I_{0}$ is normalized to the saturation intensity $I_{\text {sat }}=\hbar \omega_{0} /\left(\tau_{c} \sigma_{c}\right)$, where $\hbar \omega_{0}$ is the photon energy $\left(2 \times 10^{-19}\right.$ $\mathrm{J}$ for $977 \mathrm{~nm}$ light beam), and $\tau_{c}$ is the free carrier relaxation time, which ranges from a few picoseconds for GO aqueous dispersions [6] to a hundred of picoseconds for GO thin films [21]. The free carrier absorption cross-section $\sigma_{c}$ for GO thin films is in the range of $10^{-13}$ to $10^{-14} \mathrm{~cm}^{2}$ [21]. Within our experimental conditions, the phase shifts we observed cannot be induced by reversible saturation absorption for two reasons. First, our laser beam intensity $\left(<1 \mathrm{~W} / \mathrm{cm}^{2}\right)$ is much lower than the saturation intensity $\left(I_{\text {sat }} \simeq 0.1 \mathrm{MW} / \mathrm{cm}^{2}\right)$, so that $I_{0} / I_{\text {sat }} \simeq 10^{-5}$. Second, and more importantly, the free carrier relaxation time is much shorter than the modulation period in our experiments, i.e. $f_{m} \ll 1 /\left(2 \pi \tau_{c}\right) \simeq \mathrm{GHz}$. With the typical modulation frequencies used in our experiments $\left(f_{m} \simeq 10 \mathrm{~Hz}\right)$, the Lorentzian function in Eq. (1) takes a very low value, i.e. $2 \pi f_{m} \tau_{c} /\left(1+\left(2 \pi f_{m} \tau_{c}\right)^{2}\right) \simeq 6 \times 10^{-9}$. Based on these results, we conclude that time delays due to saturable absorption would be only measurable for modulation frequencies in the $\mathrm{GHz}$ range.

\section{The effect of the induced thermal lens}

To explain the phase shifts observed in our system we focus our attention on the thermal lensing phenomenon. The thermal lens effect depends on the thermo-physical properties of the sample [22], and it has been used in many different applications [23-25]. When the aqueous GO dispersion is excited by the laser beam a temperature gradient between the center 
of the beam and the bulk is induced, which causes a transverse gradient of the refractive index. Therefore, a thermal lens is induced in the aqueous GO dispersion. This effect can be described by assuming that the index of refraction $n$ varies with temperature according to

$$
n=n_{0}-\frac{d n}{d T} \Delta T
$$

where $n_{0}$ is the refractive index at the initial temperature, $d n / d T$ is the thermo-optic coefficient, and $\Delta T$ is the temperature change induced in the sample by the laser beam. $\Delta T$ is a function of time $t$ and the transverse distance $r$ in the plane perpendicular to the laser propagation direction (measured from the optical axis). It can be calculated using the heat equation [26]

$$
C \rho \frac{\partial \Delta T}{\partial t}=\frac{\Delta I}{L}+\kappa \nabla^{2} \Delta T
$$

where $C \rho$ is the heat capacity per unit volume ( $C$ is the heat capacity per unit mass and $\rho$ is the medium density), $\kappa$ is the thermal conductivity. The source term $\Delta I / L$ in Eq. (3) stands for the energy absorbed in the sample per unit of time and volume. The laser intensity changes along the propagation direction and can be estimated to be given by $\Delta I \propto I\left(1-e^{-\alpha L}\right)$, where $I$ is the input beam intensity, $\alpha$ is the linear absorption coefficient of the sample, and $L$ is the cuvette length. For a Gaussian beam of radius $w_{0}$ the source term in Eq. (3) reduces to $\left(2 P /\left(\pi w_{0}^{2}\right)\right) e^{-2 r^{2} / w_{0}^{2}}\left(1-e^{-\alpha L}\right) / L$, where $P$ is the input beam power. By solving Eq. (3) in the steady state and assuming the parabolic approximation (parabolic shape of $\Delta T$ near the beam center) the laser-induced thermal lens has an inverse focal length (dioptric power) given by [27]:

$$
f^{-1}=\frac{(d n / d T) P\left(1-e^{-\alpha L}\right)}{\kappa \pi w_{0}^{2}} .
$$

This expression was first derived by Gordon et al. [27] and assumes a perfect thin lens [27-29]. Later on, the aberrant nature of the thermal lens was analyzed by Sheldon et al. using a diffraction integral approach [30]. Bialkowski and Chartier obtained equivalent results with a simpler method based on the calculation of cumulative electric-field phase shifts produced by a series of Gaussian refractive-index perturbations [31]. Recent works from Malacarne et al. developed a complete thermal lens model to characterize solid materials [32]. Figure 5(a) shows the predicted thermal focal length for the $0.5 \mathrm{mg} / \mathrm{mL}$ GO water dispersion as a function of the input power. We used the thermal conductivity and the thermo-optic coefficient of water [33], i.e., $\kappa=0.6 \mathrm{~W} /(\mathrm{m} \mathrm{K})$ and $d n / d T=-0.45 \times 10^{-4} \mathrm{~K}^{-1}$, 
a beam radius of $w_{0}=1.55 \mathrm{~mm}$, the experimentally measured linear absorption coefficient $\alpha=1.77 \mathrm{~cm}^{-1}$ for the $0.5 \mathrm{mg} / \mathrm{mL}$ GO dispersion, and $L=1 \mathrm{~cm}$. The focal lengths found are close to a few meters. The temperature change in the dispersion $\Delta T$ is in the order of one kelvin. We can estimate the thermal nonlinear refractive index of the dispersion as $n_{2}=(d n / d T) w_{0}^{2}\left(1-e^{-\alpha L}\right) /(2 \kappa L) \simeq-3 \times 10^{-5} \mathrm{~cm}^{2} / \mathrm{W}$.
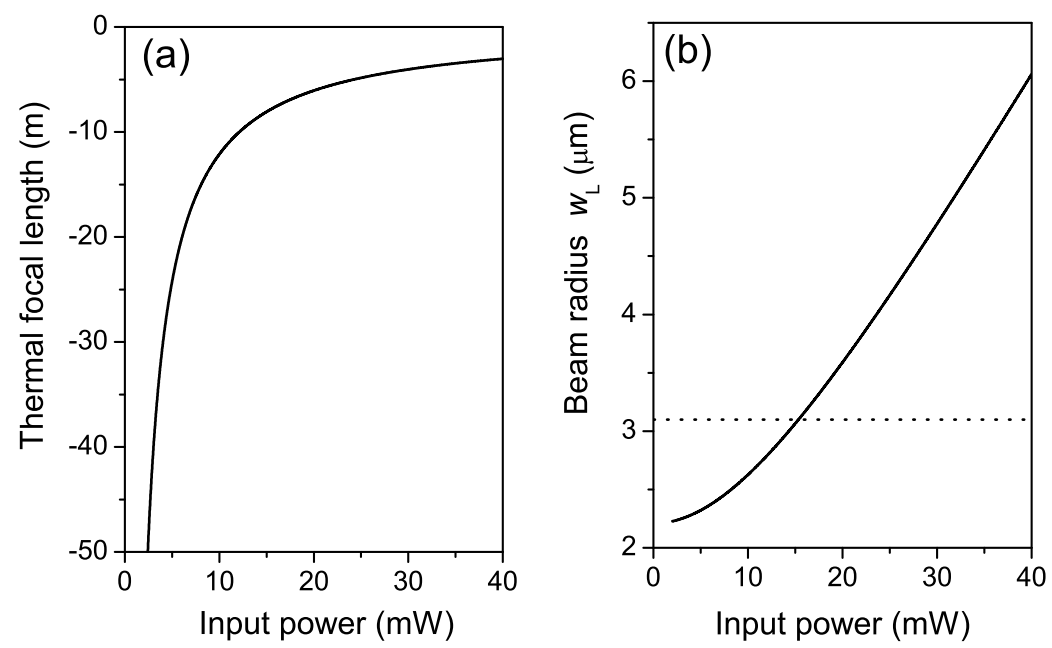

Fig. 5. (a) Thermal focal length (from Eq. (4)) versus input power. (b) Spot size at the entrance plane of the single mode fiber (from Eq. (5)) versus input power. The dotted line indicates the radius of the fiber mode $w_{f}$. We used the following parameters: $\kappa=0.6 \mathrm{~W} /(\mathrm{m} \mathrm{K}), d n / d T=-0.45 \times 10^{-4} \mathrm{~K}^{-1}$, $w_{0}=1.55 \mathrm{~mm}, \alpha=1.77 \mathrm{~cm}^{-1}(0.5 \mathrm{mg} / \mathrm{mL}$ GO water dispersion $), L=1 \mathrm{~cm}$, $d_{L}=11 \mathrm{~mm}$, and $\lambda=977 \mathrm{~nm}$.

We theoretically studied the propagation of the beam through the optical system (see Fig. $1(\mathrm{a})$ ). We used the ray transfer matrices formalism to describe the propagation of a Gaussian beam through our system [34] (in the paraxial wave approximation). The cuvette was placed at the center of the U-bench being the free space distance behind it around $d=25 \mathrm{~mm}$. We considered that a quasi-planar wavefront Gaussian beam with wavelength $\lambda=977 \mathrm{~nm}$, spot size $w_{0} \simeq 1.55 \mathrm{~mm}$ and radius of curvature $\simeq \infty$ impinges on the cuvette. This beam is partially absorbed in the sample which produces a thermal lens whose focal length is $f$ [see Eq. (4)]. This thermal lens modifies the curvature of the beam wavefront at the output of the cuvette. The new radius of curvature is $\simeq-f$. At the entrance of the focusing lens L2 the beam is nearly the same as the one at the output of the cuvette since it only propagates a distance $d \ll f$ in free space, i.e., the beam size is $\simeq w_{0}(1-d / f) \simeq w_{0}$ and the radius 
of curvature is $\simeq-f$. The coupling lens L2 focuses the beam into the output single mode fiber. We considered a focal length of $\mathrm{L} 2$ of $f_{L}=d_{L}=11 \mathrm{~mm}$. This value was estimated by using the numerical aperture of the single mode fiber $(\mathrm{NA}=0.14)$ and the typical spot size $w_{0}$, i.e., $\mathrm{NA} \simeq w_{0} / d_{L}$. At the entrance plane of the single mode fiber the beam size is

$$
w_{L}=w_{0} d_{L} \sqrt{\left(\frac{1}{f}\right)^{2}+\left(\frac{\lambda}{\pi w_{0}^{2}}\right)^{2}} .
$$

In the absence of thermal lensing, in Eq. (5) we can neglect the first term of the r.h.s $(1 / f)$, then the spot size at the entrance of output fiber is $w_{L} \simeq d_{L} \lambda /\left(\pi w_{0}\right)$, which is the focal length times the divergence of the beam $\theta \simeq \lambda /\left(\pi w_{0}\right)$. We plot in Fig. 5 (b) the beam radius at the entrance of the output fiber $w_{L}$ as a function of the input power by using the thermal focal length shown in Fig. 5(a). The spot size increases with input power. As this Gaussian beam is launched into the single mode fiber, the variation of the spot size with power can lead to a decrease of the transmittance. Efficient launching requires that the light arriving to the fiber has a complex amplitude profile similar to that of the guided mode. Then, the coupling efficiency for matching the focused beam mode to the mode of the fiber should be taken into account [35]

$$
T^{M M}=\left(\frac{2 w_{f} w_{L}}{w_{L}^{2}+w_{f}^{2}}\right)^{2}
$$

where $w_{f}$ is the radius for the fiber mode, and $T^{M M}$ represents the losses due to mode mismatch. This expression measures the square of the correlation between the intensity distribution of the Gaussian beam at the fiber entrance and the one for the guided mode of the fiber. Then, the output power can be written as $P^{\text {out }}=e^{-\alpha L} T^{\text {cuvette }} T^{M M} T^{0} P$, where $T^{0}$ accounts for the losses of the optical system. In Fig. 4(a) we plotted the simulated output-input power curves for the three GO dispersions in the experiments. We used the corresponding effective linear absorption coefficient $\alpha_{\text {eff }}$ previously obtained for each GO concentration. We consider a radius for the fiber mode $w_{f}=3.1 \mu \mathrm{m}$ and $T^{0}=0.5$. The rest of parameters are the same as in Fig. 5. The simulated curves (solid lines) agree with the experimental results (symbols). Therefore, the OL behavior found in our experiments can be attributed undoubtedly to a thermally induced nonlinear refraction.

\section{A. Thermal lens focal length oscillation}

To theoretically analyze the phase shift of the amplitude modulated beam we must take into account that the thermal lens formation takes a finite time to build up. The characteristic 
time $\tau$ depends on the thermal properties of the medium and it is usually in the range between millisecond and tenths of second. We resort again to the heat equation (3)

$$
\frac{\partial \Delta T}{\partial t_{d}}=\frac{2 P\left(1-e^{-\alpha L}\right)}{\pi \kappa L} e^{-2 r_{d}^{2}}+\nabla^{2} \Delta T,
$$

where $t_{d}=t / \tau$ is a dimensionless time normalized to $\tau=C \rho w_{0}^{2} / \kappa$ and $r_{d}=r / w_{0}$ is a dimensionless radius normalized to $w_{0}$. The modulation of the amplitude of the light beam will produce a time varying temperature spatial profile which in turn will result in an oscillating behavior of the focal length of the sample. We consider that the beam power changes in time according to: $P=P_{0}+P_{m} \cos \left(\omega_{m} t_{d}\right)$, where $P_{0}$ is the average power, $P_{m}$ is the modulation amplitude, and $\omega_{m}=2 \pi f_{m} \tau$ is the normalized (dimensionless) modulation angular frequency. Let us assume that the temperature change $\Delta T$ will be forced to oscillate at the same frequency $\Delta T=\Delta T_{0}+\Delta T_{c} \cos \left(\omega_{m} t_{d}\right)+\Delta T_{s} \sin \left(\omega_{m} t_{d}\right)$. We are considering here that the temperature change oscillation may not be in phase with the oscillation of the beam power due to the finite time response of the thermal lens. The time evolution of $\Delta T$ will produce an oscillation of the thermal lens dioptric power $D=D_{0}+D_{c} \cos \left(\omega_{m} t_{d}\right)+$ $D_{s} \sin \left(\omega_{m} t_{d}\right)$. The steady state value $\left(\Delta T_{0}\right)$ and the amplitude of the temperature change oscillation $\left(\Delta T_{c}, \Delta T_{s}\right)$ are easily obtained from the heat equation (7)

$$
\begin{aligned}
0 & =\frac{2 P_{0}\left(1-e^{-\alpha L}\right)}{\pi \kappa L} e^{-2 r_{d}^{2}}+\nabla^{2} \Delta T_{0}, \\
-\omega_{m} \Delta T_{c} & =\nabla^{2} \Delta T_{s}, \\
\omega_{m} \Delta T_{s} & =\frac{2 P_{m}\left(1-e^{-\alpha L}\right)}{\pi \kappa L} e^{-2 r_{d}^{2}}+\nabla^{2} \Delta T_{c} .
\end{aligned}
$$

The first equation (Eq. (8)) leads, in the parabolic approximation, to the well-known focal length given by Eq. (4) [27-29]. Then, the average thermal lens dioptric power $D_{0}$ is related to the average power $P_{0}$

$$
D_{0}=\frac{(d n / d T) P_{0}\left(1-e^{-\alpha L}\right)}{\kappa \pi w_{0}^{2}} .
$$

To obtain the amplitude of the temperature change oscillation $\left(\Delta T_{c}, \Delta T_{s}\right)$ we resort to Fourier space

$$
\begin{aligned}
-\omega_{m} \widehat{\Delta T}_{c} & =-(2 \pi q)^{2} \widehat{\Delta T}_{s} \\
\omega_{m} \widehat{\Delta T}_{s} & =\frac{2 P_{m}\left(1-e^{-\alpha L}\right)}{\pi \kappa L} \frac{\pi}{2} e^{-\frac{1}{2} \pi^{2} q^{2}}-(2 \pi q)^{2} \widehat{\Delta T}_{c}
\end{aligned}
$$


where $\widehat{\Delta T}_{c}$ and $\widehat{\Delta T}_{s}$ are the Fourier transforms of $\Delta T_{c}$ and $\Delta T_{s}$, respectively, and $q$ is the wavenumber (normalized to $1 / w_{0}$ ). From Eqs. (12)-(13) we obtain the Fourier amplitude of the temperature change oscillation

$$
\begin{aligned}
& \widehat{\Delta T}_{c}=\frac{(2 \pi q)^{2}}{\omega_{m}^{2}+(2 \pi q)^{4}} \frac{2 P_{m}\left(1-e^{-\alpha L}\right)}{\pi \kappa L} \frac{\pi}{2} e^{-\frac{1}{2} \pi^{2} q^{2}}, \\
& \widehat{\Delta T}_{s}=\frac{\omega_{m}}{\omega_{m}^{2}+(2 \pi q)^{4}} \frac{2 P_{m}\left(1-e^{-\alpha L}\right)}{\pi \kappa L} \frac{\pi}{2} e^{-\frac{1}{2} \pi^{2} q^{2}} .
\end{aligned}
$$

We numerically compute the inverse Fourier transform of Eqs. (14)-(15) by means of a Hankel transform

$$
\Delta T_{l}=2 \pi \int_{0}^{\infty} d q \widehat{\Delta T}_{l} J_{0}\left(2 \pi q r_{d}\right) q, \quad(l=c, s)
$$

where $J_{0}$ is the Bessel function of first kind of zero order. Then, we expand the obtained spatial profile $\Delta T_{c}$ and $\Delta T_{s}$ in the proximity of the beam center (from the center to half of the beam radius, i.e. $\left.r_{d} \simeq[0,0.5]\right)$ to a polynomial series of even powers of $r_{d}$. By assuming the parabolic approximation, we only keep the second order term of the expansion to compute the amplitude of the thermal lens dioptric power oscillation

$$
D_{l}=-\frac{2 L(d n / d T)}{w_{0}^{2}} \frac{\Delta T_{l}}{r_{d}^{2}}, \quad(l=c, s) .
$$

The thermal lens focal length oscillation leads to an oscillation of the coupling efficiency as follows: $T^{M M}=T_{0}^{M M}+T_{c}^{M M} \cos \left(\omega_{m} t_{d}\right)+T_{s}^{M M} \sin \left(\omega_{m} t_{d}\right)$, where the average transmission is

$$
T_{0}^{M M}=\left(\frac{2 w_{f} w_{L 0}}{w_{L 0}^{2}+w_{f}^{2}}\right)^{2},
$$

$w_{L 0}$ being the average beam size at the entrance plane of the output single mode fiber,

$$
w_{L 0}=w_{0} d_{L} \sqrt{D_{0}^{2}+\left(\frac{\lambda}{\pi w_{0}^{2}}\right)^{2}},
$$

and the amplitude of the transmittance oscillation is

$$
T_{l}^{M M}=\frac{4 w_{f}^{2} w_{0}^{2} d_{L}^{2}\left(w_{f}^{2}-w_{L 0}^{2}\right) 2 D_{0} D_{l}}{\left(w_{L 0}^{2}+w_{f}^{2}\right)^{3}}, \quad(l=c, s) .
$$


The output amplitude-modulated beam can be written as: $P^{\text {out }}=P_{0}^{\text {out }}+P_{m}^{\text {out }} \cos \left(\omega_{m} t_{d}-\phi\right)$, where $\phi$ accounts for the phase shift experienced by the signal beam. After some simple algebraic calculations, we obtain the phase delay $\phi$ :

$$
\phi=\frac{2 D_{0} D_{s} w_{0}^{2} d_{L}^{2}\left(w_{f}^{2}-w_{L 0}^{2}\right)}{w_{L 0}^{2}\left(w_{L 0}^{2}+w_{f}^{2}\right)} \frac{1}{\frac{P_{m}}{P_{0}}+\frac{2 D_{0} D_{c} w_{0}^{2} d_{L}^{2}\left(w_{f}^{2}-w_{L 0}^{2}\right)}{w_{L 0}^{2}\left(w_{L 0}^{2}+w_{f}^{2}\right)}}
$$

Let us compare the theoretical phase shift given by Eq. (21) with the measured phase delay. First, we analyze the case of Fig. 2, which presents the phase shift versus the modulation frequency for a $0.5 \mathrm{mg} / \mathrm{mL}$ GO water dispersion and for different input powers $P_{0}=2.5 \mathrm{~mW}$, $P_{0}=18.4 \mathrm{~mW}$, and $P_{0}=28.5 \mathrm{~mW}$. We used the same parameters as above: $w_{0} \simeq 1.55 \mathrm{~mm}$, $\kappa=0.6 \mathrm{~W} /(\mathrm{m} \mathrm{K}), d n / d T=-0.45 \times 10^{-4} \mathrm{~K}^{-1}, \alpha=1.77 \mathrm{~cm}^{-1}, f_{L}=d_{L}=11 \mathrm{~mm}, w_{f}=3.1$ $\mu \mathrm{m}$. We varied the thermal lens formation time to fit the experimental curves, obtaining $\tau=0.2 \mathrm{~s}$. The simulated phase shift curves are plotted in Fig. 2 (solid lines) and show a good agreement with the experimental findings. That is, positive or negative phase delays are achieved depending on the input power value. A transition between positive to negative delays takes place as the input power increases. However, at high input powers and low modulation frequencies the simulated curve deviates from the experimental data (see Fig. 2 ). In this regime, the experimental output signal presents some distortion (see black curve in the upper inset in Fig. 6). The shape of the output signal produces larger phase shifts than those found in the simulations. The nonlinear nature of the physical processes involved in our system could bring the generation of harmonic distortion. The spectrum of the experimental output signal shows a non negligible amplitude for the second harmonic $\left(A_{2 n d}\right)$ in comparison to the amplitude of the fundamental one $\left(A_{1 s t}\right)$ (not shown). In order to quantify the effect of the harmonic distortion we compute the distortion factor as $A_{2 n d} / \sqrt{A_{1 s t}^{2}+A_{2 n d}^{2}}$. The result is shown in Fig. 6 (symbols). Effectively, at high input powers and low modulation frequencies, the harmonic distortion becomes relevant with values above $5 \%$. This nonlinear distortion can be theoretically calculated by our model. In order to calculate the simulated output signal we retained second order terms in the modulation amplitudes $P_{m}, D_{c}$ and $D_{s}$. By computing the spectrum of the simulated output signals we obtain the distortion factor shown in Fig. 6 (solid lines), which agrees with the experimental one.

Now, we turn our attention to analyze the phase shift dependence with GO concentration. That is, we try to reproduce the experimental results of Fig. 3. We use the effective linear absorption coefficient from the slopes of Fig. 4(b): $\alpha_{\text {eff }}=0.92 \mathrm{~cm}^{-1}(0.25 \mathrm{mg} / \mathrm{mL}), \alpha_{e f f}=$ $1.77 \mathrm{~cm}^{-1}(0.5 \mathrm{mg} / \mathrm{mL})$, and $\alpha_{\text {eff }}=2.52 \mathrm{~cm}^{-1}(1 \mathrm{mg} / \mathrm{mL})$. The average input power is $P_{0}=18.4 \mathrm{~mW}$. In Fig. 3 we plot the simulated phase shift curves (solid lines), which roughly agree with the experimental ones. Furthermore, we analyze the possible variation of the 


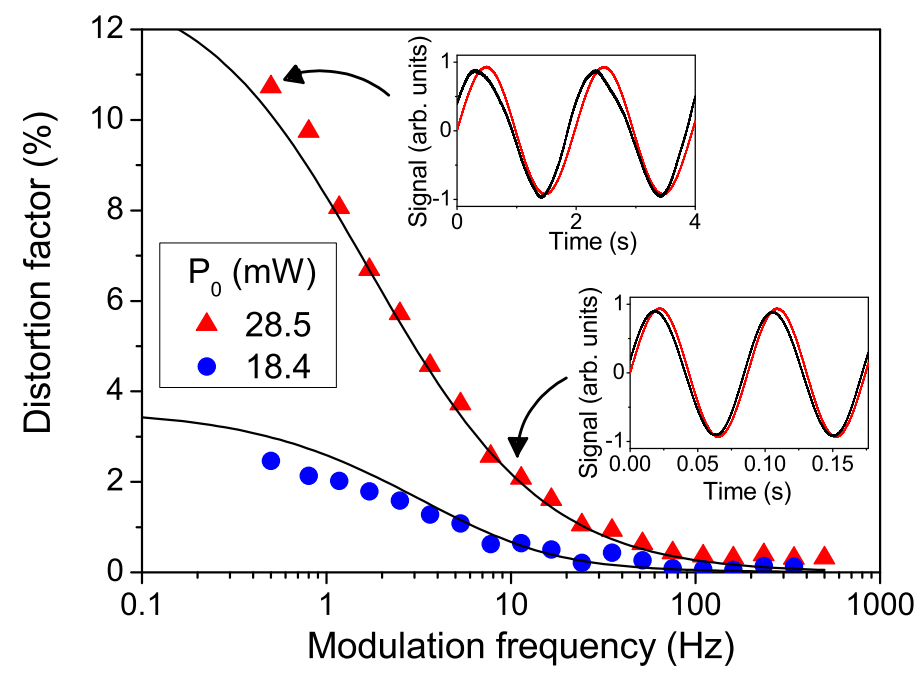

Fig. 6. Experimental (symbols) and theoretical (lines) distortion factor versus modulation frequency for a $0.5 \mathrm{mg} / \mathrm{mL}$ GO water dispersion and different input powers: $P_{0}=18.4 \mathrm{~mW}$ (circles), $P_{0}=28.5 \mathrm{~mW}$ (triangles). In the insets, time evolution of both, the reference signal (red line) and the signal propagated through the GO dispersion and the U-bench (black line) for the modulation frequencies marked with the arrows.

thermal conductivity with the concentration of GO. The volume fraction of GO changes with concentration as: $\phi_{v}=0.0032(0.25 \mathrm{mg} / \mathrm{mL}), \phi_{v}=0.0065(0.5 \mathrm{mg} / \mathrm{mL})$, and $\phi_{v}=0.013(1$ $\mathrm{mg} / \mathrm{mL}$ ). As a first approach to this question we used the Maxwell-Garnet effective medium theory to estimate an effective thermal conductivity [36] $\kappa_{\text {eff }} \simeq \kappa_{\text {water }}\left(1+2 \phi_{v}\right) /\left(1-\phi_{v}\right)$. In this expression, we assumed that the thermal conductivity of the GO sheets is much larger than the thermal conductivity of water $\left(\kappa_{\text {water }}\right)$. Using $\kappa_{\text {water }}=0.59 \mathrm{~W} /(\mathrm{m} \mathrm{K})[33]$, we obtained the following effective thermal conductivities: $\kappa_{\text {eff }}=0.596 \mathrm{~W} /(\mathrm{m} \mathrm{K})(0.25$ $\mathrm{mg} / \mathrm{mL}), \kappa_{\text {eff }}=0.6 \mathrm{~W} /(\mathrm{m} \mathrm{K})(0.5 \mathrm{mg} / \mathrm{mL})$, and $\kappa_{\text {eff }}=0.613 \mathrm{~W} /(\mathrm{m} \mathrm{K})(1 \mathrm{mg} / \mathrm{mL})$. As a results, the lowest $(0.25 \mathrm{mg} / \mathrm{mL})$ and highest $(1 \mathrm{mg} / \mathrm{mL})$ concentration samples have a slightly different thermal conductivity than the one used in our simulations (solid lines in Fig. 3). Taking into account this thermal conductivity dependence with concentration, we plot in Fig. 3 (with dashed lines) the two new simulated phase shift curves which present a closest agreement with the experimental results. In summary, the behavior of the phase shift in our system is well explained by the interplay between the thermal lens focal length oscillation induced in the water GO dispersion and the mode matching coupling in the single 
mode fiber.

Finally, with the aim of obtaining an analytical expression for the phase shift that explicitly contains the modulation frequency dependence, we assume that the time evolution of the dioptric power $D$ of the thermal lens follows an exponential decay

$$
\frac{\partial D}{\partial t_{d}}=-D+D^{s t}
$$

where $D^{s t}$ is the stationary value of the dioptric power, which is equal to the inverse of the focal length of the thermal lens given by Eq. (4). When we modulate the beam power $\left[P=P_{0}+P_{m} \cos \left(\omega_{m} t_{d}\right)\right]$, we can easily obtain the amplitude of the dioptric power oscillation

$$
\begin{aligned}
& D_{c}=\frac{1}{1+\omega_{m}^{2}} D_{0} \frac{P_{m}}{P_{0}}, \\
& D_{s}=\frac{\omega_{m}}{1+\omega_{m}^{2}} D_{0} \frac{P_{m}}{P_{0}} .
\end{aligned}
$$

Using these expressions (23)-(24) in the phase shift presented above [see Eq. (21)], we obtain the analytical dependence of the phase shift with the modulation frequency $\omega_{m}=$ $2 \pi f_{m} \tau$

$$
\phi=\frac{2 D_{0}^{2} w_{0}^{2} d_{L}^{2}\left(w_{f}^{2}-w_{L 0}^{2}\right)}{w_{L 0}^{2}\left(w_{L 0}^{2}+w_{f}^{2}\right)} \frac{2 \pi f_{m} \tau}{1+\frac{2 D_{0}^{2} w_{0}^{2} d_{L}^{2}\left(w_{f}^{2}-w_{L 0}^{2}\right)}{w_{L 0}^{2}\left(w_{L 0}^{2}+w_{f}^{2}\right)}+\left(2 \pi f_{m} \tau\right)^{2}} .
$$

This expression tells us that a positive or negative phase shift is obtained depending on whether the average beam size $w_{L 0}$ is smaller or greater than the fiber mode size $w_{f}$. It can be seen from Eq. (25) that the maximum phase shift is achieved at the modulation frequency $f_{m}=f_{\text {max }}$ :

$$
f_{\max }=\frac{1}{2 \pi \tau} \sqrt{1+\frac{2 D_{0}^{2} w_{0}^{2} d_{L}^{2}\left(w_{f}^{2}-w_{L 0}^{2}\right)}{w_{L 0}^{2}\left(w_{L 0}^{2}+w_{f}^{2}\right)}} .
$$

The value of this modulation frequency is roughly given by the characteristic thermal lens formation time $f_{\max } \simeq 1 /(2 \pi \tau)$. For $\tau$ in the tenths of seconds range, we obtain a few Hertz frequency, in agreement with the experimental results (see Figs. 2 and 3). Furthermore, in agreement with the experiments (see Fig. 2), the optimum frequency (Eq. (26)) is power dependent, since it varies with the average dioptric power $D_{0}$, which in turn is proportional to $P_{0}$. 


\section{Conclusions}

We analyzed the phase shift induced in an amplitude-modulated light beam when propagating through GO water dispersions in a fiber-to-fiber U-bench. We observed an increase of the phase shift with GO concentration. Positive phase shifts are achieved for low input powers whereas higher input powers lead to negative phase shifts. Transmittance measurements show that the system exhibits a strong optical limiting response. A simple theoretical model reveals that the phase shift arises from the thermal lens focal length oscillation induced in the GO dispersion and the mode matching coupling in the single mode output fiber. We have shown that time delays due to GO saturable absorption or free carrier absorption would be only measurable in our system for modulation frequencies in the GHz range.

Ultrawide broadband and strong light absorption properties of GO renders it an excellent material for optical limiting applications. The findings of this work can be extended to other graphene families, and also in applications such as biosensing using infrared radiation [15].

\section{Acknowledgments}

This work has been supported by projects FIS2010-22082 (Ministerio de Ciencia e Innovación, MICINN), MAT2009-09335 (Ministerio de Economía y Competitividad, MINECO). H.J.S. thanks MINECO for a Ramón y Cajal Research Fellow.

\section{References}

1. D. R. Dreyer, S. Park, C. W. Bielawski, and R. S. Ruoff, "The chemistry of graphene oxide," Chem. Soc. Rev. 39, 228-240 (2010).

2. K. P. Loh, Q. Bao, G. Eda, and M. Chhowalla, "Graphene oxide as a chemically tunable platform for optical applications," Nature Chemistry 2, 1015-1024 (2010).

3. M. Hirata, T. Gotou, S. Horiuchi, M. Fujiwara, and M. Ohba, "Thin-film particles of graphite oxide 1: High-yield synthesis and flexibility of the particles," Carbon 42, 29292937 (2004).

4. J. I. Paredes, S. Villar-Rodil, A. Martínez-Alonso, and J. M. D. Tascón, "Graphene oxide dispersions in organic solvents," Langmuir 24, 10560-1564 (2008).

5. S. Park and R. S. Ruoff, "Chemical methods for the production of graphenes," Nat. Nanotechnol. 4, 217-224 (2009).

6. S. Kumar, M. Anija, N. Kamaraju, K. S. Vasu, K. S. Subrahmanyam, A. K. Sood, and C. N. R. Rao, "Femtosecond carrier dynamics and saturable absorption in graphene suspensions," Appl. Phys. Lett. 95, 191911 (2009).

7. Z. Liu, Y. Wang, X. Zhang, Y. Xu, Y. Chen, and J. Tian, "Optical properties of graphene oxide in nanosecond and picosecond regimes," Appl. Phys. Lett. 94, 021902 (2009). 
8. J. Wang, Y. Hernandez, M. Lotya, J. N. Coleman, and W. J. Blau, "Broadband nonlinear optical response of graphene dispersions," Adv. Mater. 21, 2430-2435 (2009).

9. M. Feng, H. Zhan, and Y. Chen, "Nonlinear optical and optical limiting properties of graphene families," Appl. Phys. Lett. 96, 033107 (2010).

10. N. Liaros, P. Aloukos, A. Kolokithas-Ntoukas, A. Bakandritsos, T. Szabo, R. Zboril, and S. Couris, "Nonlinear optical properties and broadband optical power limiting action of graphene oxide colloids," J. Phys. Chem. C 117, 6842-6850 (2013).

11. Y. Xu, Z. Liu, X. Zhang, Y. Wang, J. Tian, Y. Huang, Y. Ma, X. Zhang, and Y. Chen, "A graphene hybrid material covalently functionalized with porphyrin: synthesis and optical limiting property," Adv. Mater. 21, 1275-1279 (2009) .

12. R. Wu, Y. Zhang, S. Yan, F. Bian, W. Wang, X. Bai, X. Lu, J. Zhao, and E. Wang, "Purely coherent nonlinear optical response in solution dispersions of graphene sheets," Nano Lett. 11, 5159-5164 (2011).

13. X-L. Zhang, Z-B. Liu, X-C. Li, Q. Ma, X-D. Chen, J-G. Tian, Y-F. Xu, and Y-S. Chen, "Transient thermal effect, nonlinear refraction and nonlinear absorption properties of graphene oxide sheets in dispersion," Opt. Express 21, 7511-7520 (2013).

14. J. Li, Y. Zhang, H. Li, C. Yao, and P. Yuan, "Observation of tunable superluminal propagation in the single-layer graphene oxide solution", Opt. Comm. 295, 226-229 (2013).

15. C. Liu, Z. Wang, H. Jia, and Z. Li, "Efficient fluorescence resonance energy transfer between upconversion nanophosphors and graphene oxide: a highly sensitive biosensing platform," Chem. Commun. 47, 46614663 (2011).

16. X. Zhao, Z-B. Liu, W-B. Yan, Y. Wu, X-L. Zhang, Y. Chen, and J-G. Tian, "Ultrafast carrier dynamics and saturable absorption of solution-processable few-layered graphene oxide," Appl. Phys. Lett. 98, 121905 (2011).

17. M. S. Bigelow, N. N. Lepeshkin, and R. W. Boyd, "Superluminal and slow light propagation in a room-temperature solid," Science 301, 200-202 (2003).

18. F. Arrieta-Yáñez, O.G. Calderón, and S. Melle, "Fast light based on excited-state absorption in erbium doped fibers," in IONS 9 International OSA Network of Students (Optical Society of America, 2011).

19. G. S. Agarwal and T. N. Dey, "Sub- and superluminal propagation of intense pulses in media with saturated and reverse absorption," Phys. Rev. Lett. 92, 203901 (2004).

20. H. Wang, Y. Zhang, N. Wang, W. Yan, H. Tian, W. Qiu, and P. Yuan, "Observation of superluminal propagation at negative group velocity in $\mathrm{C}_{60}$ solution, Appl. Phys. Lett. 90, 121107 (2007).

21. G. Leonard, Thesis "Free carrier absorption in graphene oxide thin film," National University of Singapore, 2012, http://www.physics.nus.edu.sg/student/Honours Projects 
Repository/leonard Goh fyp-final.pdf

22. S. E. Bialkowski. Photothermal spectroscopy methods for chemical analysis (Wiley, New York, 1996).

23. D. Rojas, R. J. Silva, J. D. Spear, R. E. Russo, "Dual-beam optical fiber thermal lens spectroscopy," Anal. Chem. 63, 1927-1932 (1991).

24. M. Franko and C. D. Tran, Encyclopedia of Analytical Chemistry: Thermal Lens Spectroscopy (2010). DOI: 10.1002/9780470027318.a9079

25. C. Estupiñán-López, C. T. Dominguez, R. de Araujo. "Eclipsing thermal lens spectroscopy for fluorescence quantum yield measurement," Opt. Express 21, 18592-18601 (2013).

26. R. W. Boyd, Nonlinear Optics (second ed., Academic Press, San Diego, California, 2003), Chap. 4.5 .

27. J. P. Gordon, R. C. C. Leite, R. S. Moore, S. P. S. Porto, and J. R. Whinnery, "Longtransient effects in lasers with inserted liquid samples," J. Appl. Phys. 36, 3-8 (1965).

28. J. R. Whinney, "Laser measurement of optical absorption in liquids," Acc. Chem. Res. 7, 225-231 (1974).

29. C. Hu and J. R. Whinnery, "New thermooptical measurement method and a comparison with other methods," Appl. Opt. 12, 72-79 (1973).

30. S. J. Sheldon, L. V. Knight, and J. M. Thorne, "Laser-induced thermal lens effect: a new theoretical model," Appl. Opt. 21, 1663-1669 (1982).

31. S.E. Bialkowski and A. Chartier, "Diffraction effects in single- and two-laser photothermal lens spectroscopy," Appl. Opt. 36, 6711-6721 (1997).

32. L.C. Malacarne, N.G.C. Astrath, L.S. Herculano, "Laser-induced wavefront distorsion in optical materials: a general model," J. Opt. Soc. Am. B 29, 3355-3359 (2012).

33. American Institute of Physics Handbook (McGraw-Hill, New York, 1957), Sections 4g and $6 \mathrm{~b}$.

34. A. E. Siegman, Lasers (Mill Valley, CA: Univ. Sci. Books, 1986).

35. A. Sennaroglu, "Effect of thermal lensing on the mode matching between pump and laser beams in $\mathrm{Cr}^{4+}$ : forsterite lasers: a numerical study," J. Phys. D: Appl. Phys. 33, 1478-1483 (2000).

36. B-X. Wang, L-P. Zhou, and X-F. Peng, "A fractal model for predicting the effective thermal conductivity of liquid with suspension of nanoparticles," Inter. J. Heat Mass Transfer 46, 2665-2672 (2003). 


\section{List of Figures}

1 (a) Experimental setup. C, cuvette containing the graphene oxide dispersion. DFB LD, distributed feedback laser diode at $977 \mathrm{~nm}$. LD TEC, laser diode temperature and current controller. FG, function generator. OC, digital oscilloscope. PD1, reference photodetector. PD2, photodetector for the GO signal. L1, collimating lens. L2, focusing lens. Green lines, single mode optical fibers. (b) Cuvette containing an aquoeus dispersion of GO sheets with a concentration of $0.25 \mathrm{mg} / \mathrm{mL}$. (c) Time evolution of the experimental reference signal (red line) and the signal propagated through the GO dispersion and the Ubench (black line) for a $0.5 \mathrm{mg} / \mathrm{mL}$ graphene oxide water dispersion, a laser input power of $P_{0}=28.5 \mathrm{~mW}$, and a modulation frequency of $f_{m}=1.71 \mathrm{~Hz}$.

2 Experimental (symbols) and theoretical (lines) phase delay versus modulation frequency for a $0.5 \mathrm{mg} / \mathrm{mL}$ GO aqueous dispersion and different input powers: $P_{0}=2.5 \mathrm{~mW}$ (squares), $P_{0}=18.4 \mathrm{~mW}$ (circles), $P_{0}=28.5 \mathrm{~mW}$ (triangles). Inset: phase delay versus input power for a modulation frequency of $f_{m}=3.64$ Hz. . . . . . . . . . . . . . . . . . . . .

3 Experimental (symbols) and theoretical (lines) phase delay versus modulation frequency for a laser input power of $18.4 \mathrm{~mW}$ and different $\mathrm{GO}$ concentrations: $0.25 \mathrm{mg} / \mathrm{mL}$ (squares), $0.5 \mathrm{mg} / \mathrm{mL}$ (circles), and $1 \mathrm{mg} / \mathrm{mL}$ (triangles). Simulated curves with a fixed value of the thermal conductivity $\kappa$ (solid lines), and changing the value of $\kappa$ according to the volume fraction of GO sheets

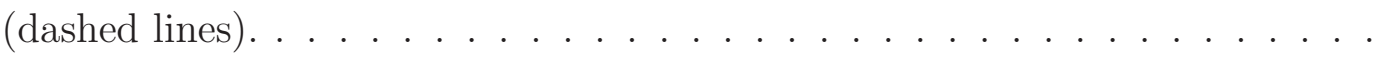

4 Experimental (symbols) output-input power curves for different GO concentrations: $0.25 \mathrm{mg} / \mathrm{mL}$ (squares), $0.5 \mathrm{mg} / \mathrm{mL}$ (circles), and $1 \mathrm{mg} / \mathrm{mL}$ (triangles). (a) Output power measured at the output optical fiber of the U-bench. Theoretical curves (solid lines). (b) Output power measured at the output of the cuvette (before the beam enters L2). The dotted lines are linear fits to the experimental data and the corresponding slopes are also indicated in panel (b).

(a) Thermal focal length (from Eq. (4)) versus input power. (b) Spot size at the entrance plane of the single mode fiber (from Eq. (5)) versus input power. The dotted line indicates the radius of the fiber mode $w_{f}$. We used the following parameters: $\kappa=0.6 \mathrm{~W} /(\mathrm{m} \mathrm{K}), d n / d T=-0.45 \times 10^{-4} \mathrm{~K}^{-1}$, $w_{0}=1.55 \mathrm{~mm}, \alpha=1.77 \mathrm{~cm}^{-1}(0.5 \mathrm{mg} / \mathrm{mL}$ GO water dispersion $), L=1 \mathrm{~cm}$, $d_{L}=11 \mathrm{~mm}$, and $\lambda=977 \mathrm{~nm}$. 
6 Experimental (symbols) and theoretical (lines) distortion factor versus modulation frequency for a $0.5 \mathrm{mg} / \mathrm{mL}$ GO water dispersion and different input powers: $P_{0}=18.4 \mathrm{~mW}$ (circles), $P_{0}=28.5 \mathrm{~mW}$ (triangles). In the insets, time evolution of both, the reference signal (red line) and the signal propagated through the GO dispersion and the U-bench (black line) for the modulation frequencies marked with the arrows. . . . . . . . . . . . . . . . . 15 